\title{
Crystal Plasticity FE Modeling of Ti Alloys for a Range of Strain-Rates. Part II: Image-based Model with Experimental Validation
}

\author{
Somnath Ghosha,*, Ahmad Shahba ${ }^{\mathrm{b}}$, Xiaohui Tu ${ }^{\mathrm{b}}$, \\ Emily L. Huskins ${ }^{c}$, Brian E. Schuster ${ }^{d}$ \\ ${ }^{a}$ M. G. Callas Professor, Departments of Civil and Mechanical Engineering, Johns \\ Hopkins University \\ ${ }^{\mathrm{b}}$ Graduate Research Assistant, Civil Engineering, Johns Hopkins University \\ ${ }^{\mathrm{c}}$ Assistant Professor, Mechanical Engineering, US Naval Academy \\ ${ }^{\mathrm{d}}$ Research Scientist, US Army Research Laboratory, RDRL-WM, Aberdeen \\ Proving Ground
}

\begin{abstract}
The second of this two-part paper develops an image-based crystal plasticity finite element model for simulating $h c p$ metals deforming at a wide of range of strainrates. It incorporates a unified flow rule based crystal plasticity constitutive model, combining the thermally-activated and drag-dominated stages of dislocation glide, proposed in part I (Shahba and Ghosh, 2016). The image-based CPFE uses 3D statistically-equivalent virtual microstructures that are constructed by stereology and statistics from 2D surface EBSD maps. The statistically equivalent virtual microstructures are constructed for the Ti-7Al alloy in as-rolled (AR) and rolledannealed (RA) conditions. The virtual microstructures are discretized into 3D tetrahedral elements that are stabilized to yield locking-free large deformation FE results. This paper demonstrates the competency of the model for simulating deformation of the polycrystalline $\mathrm{Ti}-7 \mathrm{Al}$ alloy microstructures under quasi-static and high rates of deformation. Room temperature compression tests at quasi-static $\left(10^{-3} s^{-1}\right)$ and dynamic strain rates $\left(1000-4000 s^{-1}\right)$ are performed and used to calibrate and validate the constitutive model. The simulations reveal that the decrease in the hardening rate is significant under adiabatic conditions due to the promotion of slip-driven plasticity. The effect of degradation of elastic constants with temperature on the macroscopic behavior is noticeable only at the later stages of deformation. A study on adiabatic heating reveals that grains undergoing severe plastic deformation do not necessarily yield higher temperatures. In contrast, grains that are less favorably oriented for dislocation slip could experience higher adiabatic heating due to higher local stresses.
\end{abstract}

Preprint submitted to International Journal of Plasticity

29 August 2016

(C) 2016. This manuscript version is made available under the Elsevier user license

http://www.elsevier.com/open-access/userlicense/1.0/ 
Key words: Ti-7Al alloy, image-based crystal plasticity, statistically-equivalent microstructure, thermal activation, drag, adiabatic heating

\section{Introduction}

In the first of this two-part paper, a dislocation density-based crystal plasticity constitutive relation with a unified flow rule has been developed for modeling deformation in $h c p$ Ti alloys for a wide range of strain-rates. The unified flow rule is arrived at by combining thermally-activated and dragdominated stages of dislocation slip. The second part of this paper develops image-based crystal plasticity finite element (CPFE) models that use the unified flow theory to model deformation at a range of strain-rates. Image-based models explicitly capture the morphological and crystallographic features of the material microstructure, such as grain size and shape, micro-texture and misorientation distributions, as well as micro-mechanisms responsible for deformation and failure. Once validated, large number of virtual specimens with various microstructural features can be simulated for predicting the relation between a microstructure and its response function. Significance of using realistic 3D microstructures in crystal plasticity $\mathrm{FE}$ analysis of Ti alloys has been recently highlighted in (Zhang et al., 2015). A significant benefit of these models is their comprehension and low cost in comparison with experiments on real specimens. With increasing computing power and low costs, a large number of image-based models of polycrystalline materials have been developed for simulations and property evaluation, e.g. in (Thomas et al., 2012; Clavton, 2005; Anahid et al., 2011; Cheng and Ghosh, 2015; F. et al., 2010; Roters et al., 2010; Lebensohn et al., 2012; Lebensohn, 2001; Knezevic et al., 2009) and others. Some of these models use the finite element methods, e.g. crystal plasticity FEM or CPFEM, for numerical simulations, while others use the efficient fast Fourier transform (FFT) using the Green's function method (Lebensohn et al., 2012; Lebensohn, 2001; Knezevic et al., 2009).

Developing robust image-based CPFE models for simulating deformation leading to fatigue and failure involves a number of steps. The first step is the creation of statistically equivalent representative volume elements or SERVEs of the material microstructure accounting for the morphology and crystallography from orientation imaging microscopy (OIM) or electron back-scattered

\footnotetext{
* Corresponding author: 3400 N. Charles St., Baltimore, MD 21218, Tel: +1-410516-7833; fax: +1-410-516-7473

Email address: sghosh20@jhu.edu (Somnath Ghosh).
} 
diffraction (EBSD) images of the microstructure. Reconstruction of statistically equivalent virtual polycrystalline microstructures from 2D or 3D EBSD data have been developed in (Groeber et al., 2008a,b); Groeber and Jackson, 2014) by matching morphological and crystallographic statistics. The next step in the CPFEM development involves generation of conforming mesh for these polycrystalline SERVEs. Discretization of these complex microstructures with sharp grain boundaries and multiple grain junctions, is best accomplished using 3D four-node tetrahedral or TET4 elements. These elements can be made to conform to the complex geometries using sophisticated meshing codes and software products e.g. (Simulation Modeling Suite, 2015). However these elements suffer from severe volumetric locking when simulating deformation using nearly-incompressible crystal plasticity constitutive relations. This can give rise to large spurious stresses in the computational models, often leading to premature failure predictions. Recently a locking-free stabilized TET4 element that uses a F-bar patch, has been developed for effective CPFE analysis in (Cheng et al., 2016). Finally, the incorporation of robust constitutive models for accurate material response prediction is necessary in CPFE modeling. The physics-based unified CP constitutive model, developed in part I (Shahba and Ghosh, 2016), has unique attributes of simulating a range of deformation rates through a smooth transition from thermally-activated to drag drag-dominated processes and vice-versa.

The part II develops an image based crystal plasticity FE model for the single-phase Ti alloy Ti-7.02Al-0.11O-0.015Fe (wt\%) or Ti-7Al. Two different microstructures, emanating from different processing conditions are considered. The model parameters are calibrated from experiments conducted at a range of strain-rates. Furthermore the image-based model is validated using results from these tests. The calibrated models is then used to study the effect of temperature on the mechanical response under isothermal and adiabatic conditions. Inertia effects are neglected in this study, since it is focused on constitutive modeling. However, macroscopic simulations at high rates using response relations in this model can implement inertial effects for modeling phenomena such as dynamic stress waves.

The paper is organized as follows. Section 2 discusses the materials and explains the procedure of reconstructing statistically equivalent microstructures from collected 2D data. This section concludes with an FE mesh convergence study. Section 3 provides the details of the quasi-static and dynamic tests performed for calibration and validation of the constitutive models. Section 4 discusses the procedure of calibration and validation of the constitutive models. Numerical results are provided which highlight the importance of temperature in the context of isothermal and adiabatic simulations. Concluding remarks are made in Section 6 . 


\section{Microstructural Characterization and Statistically Equivalent Rep- resentative Volume Elements}

This section briefly discusses the material characterization and reconstruction methods for 3D virtual microstructures from 2D measurements. The material studied in this paper is Ti-7.02Al-0.11O-0.015Fe (wt\%), better known as Ti-7Al alloy, with a predominantly hcp crystallographic structure Brandes, 2008). The composition of this alloy is very close to the $\alpha$ phase of many commercially available titanium alloys (Pilchak, 2013). Mechanical testing is done on two variants of this alloy, based on their pre-test processing. They are the purely rolled (AR) samples and the rolled-annealed (RA) samples, which are first rolled and subsequently annealed to increase the grain size and improve its ductility.

\subsection{Microstructural Characterization}

Ti-7Al specimens are imaged using electron back-scattered diffraction (EBSD) in a scanning electron microscopy system, provided in (Pilchak, 2015). For the AR and RA samples, surface EBSD scans quantifying the texture are respectively collected on regions with dimension $5425 \times 2190 \mu m^{2}$ and $5175 \times$ $2135 \mu \mathrm{m}^{2}$, at $5 \mu \mathrm{m}$ step size. A portion of the surface EBSD scans for both the samples are shown in figure 1. The scans have been filtered to remove noise from the data based on a confidence index. The pole figures for the $\{0001\}$ and $\{\overline{2} 110\}$ orientations in figure 1 indicate that the crystallographic $c$-axis of grains is mostly aligned with the normal direction (ND) for both the AR and RA microstructures. The most significant morphological difference between these two sample microstructures is the grain size due to the annealing process. The averaged diameters of the equivalent 2D circular grains are $34.9 \mu \mathrm{m}$ and $83.4 \mu m$, for the AR and RA samples respectively.

\subsection{Generating Virtual Microstructures}

Methods of reconstructing 3D virtual microstructures have been developed using techniques like serial-sectioning using focused ion beam (Ghosh et al., 2008; Bhandari et al., 2007; Groeber et al., 2007), manual polishing (Rowenhorst and Voorhees, 2005) and X-ray tomography (Lauridsen et al., 2006). However, these methods need 3D data for microstructure reconstruction. In the present work, 3D statistics are estimated from extrapolation of 2D surface EBSD data on polycrystalline specimens as done in (Thomas et al., 2012) for other alloys. The 2D EBSD images for both samples in figure 1 are characterized. Crystallographic 


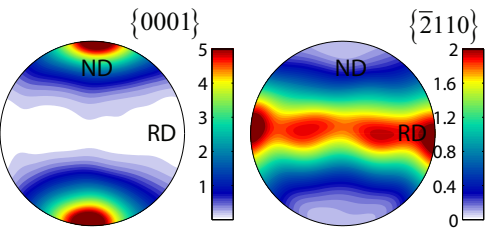

$1 \mathrm{~mm}$

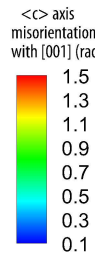

(a)
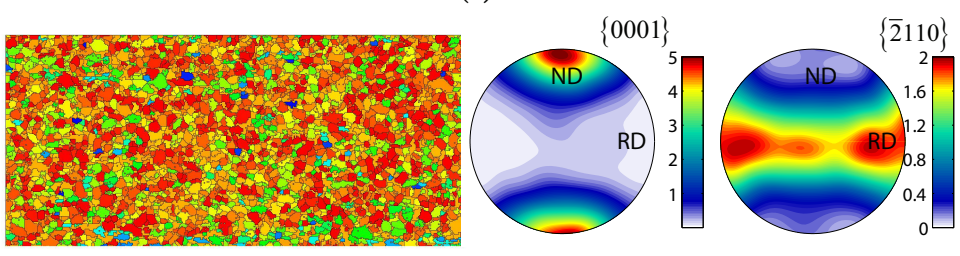

$1 \mathrm{~mm}$

(b)

Fig. 1. Filtered EBSD scans and pole figures for the (a) RA and (b) AR samples

distributions, e.g. orientation and misorientation distributions, and morphological distribution, e.g. distribution of 2D equivalent circular grain diameters $(E C D)$, are obtained. To generate 3D statistics from $2 \mathrm{D}$ distributions, a procedure based on stereology principles in (Russ and Dehoff, 1999) is employed. Assuming equiaxed grains, the average equivalent spherical grain diameter $(\overline{E S D})$ is expressed in terms of the average equivalent grain diameter $\overline{E C D}$ as:

$$
\overline{E S D}=\frac{4}{\pi} \overline{E C D}
$$

The standard deviation of the 2D and 3D grain idealizations, $C^{s d}$ and $S^{s d}$ respectively, are assumed to be the same. The averaged 3D grain diameters $\overline{E S D}$ and its standard deviation $S^{s d}$, calculated from the 2D distributions in the images of figure 1 are given in Table 1 for both the AR and RA samples.

The log-normal probability density function has been found to reasonably

\section{Table 1}

\begin{tabular}{|c|c|c|}
\hline Microstructure (Process) & $\overline{E S D}(\mu \mathrm{m})$ & $S^{s d}(\mu \mathrm{m})$ \\
\hline Annealed (AR) & 44.4 & 15.4 \\
Rolled Annealed (RA) & 106.2 & 51.7 \\
\hline
\end{tabular}

Average and standard deviation of the $3 \mathrm{D}$ equivalent grain diameter for RA and AR samples

represent the grain size distribution in metallic alloys such as Ni-based superalloys in (Groeber et al., 2008a) and Ti alloys in (Thomas et al., 2012). This distribution function is defined in terms of two parameters viz. the average $\mu$ and standard deviation $\sigma$ of the population. They can be computed in terms of the grain size average and standard deviation values in table 1 using the 
following formulae given in (Mood et al., 1974):

$$
\begin{aligned}
& \mu=\ln \left({\overline{E S D^{2}}}^{2} / \sqrt{S^{s d^{2}}+{\overline{E S D^{2}}}^{2}}\right) \\
& \sigma=\sqrt{\ln \left[\left(\frac{S^{s d}}{\overline{E S D}}\right)^{2}+1\right]}
\end{aligned}
$$

Alternatively one can employ the method of maximum-likelihood to obtain the parameters of the log-normal distribution.

The morphological and crystallographic orientation, misorientation and microtexture distribution data are input into the DREAM.3D software (Groeber and Jackson, 2014) to create 3D statistically-equivalent virtual microstructures using methods described in (Groeber et al., 2008a, b) . For generating synthetic microstructures, this software relaxes the assumption of equiaxed grains used in the development of morphological statistics. Grains are allowed to have complex shapes with random morphological orientations. These grains are then packed in the synthetic microstructure based on neighborhood constraints. Following previous reconstructions for Ti alloys in (Thomas et al., 2012) and IN100 superalloys in (Groeber et al., 2008a), the average number of neighbors to a grain in the 3D ensemble is assumed to be 14. Crystallographic orientations are assigned to the grains in the synthetic microstructure by sampling from the experimentally obtained orientation distribution function. Subsequently, the misorientation and micro-texture distributions are matched by an error minimization routine, in which orientations are swapped among grains and/or perturbed. A total of 180 different synthetic microstructures with varying number of grains are reconstructed for the RA sample. These microstructures are grouped into different bins based on the number of grains in the ensemble. Each bin has a width of 10, i.e. the increase in the number of grains for every bin is by 10. Error measures for the average grain size and the standard deviation are defined to quantitatively study the convergence in grain size distribution. They are:

$$
\begin{aligned}
& E_{a v}^{(i)}=\frac{1}{n^{(i)}} \sqrt{\sum_{j=1}^{n^{(i)}}\left(\frac{\overline{E S D_{j}^{(i)}}}{\overline{E S D}}-1\right)^{2}} \\
& E_{s d}^{(i)}=\frac{1}{n^{(i)}} \sqrt{\sum_{j=1}^{n^{(i)}}\left(\frac{S^{s d^{(i)}}}{S^{s d}}-1\right)^{2}}
\end{aligned}
$$

For a microstructures in the $i$ th bin, $E_{a v}^{(i)}$ and $E_{s d}^{(i)}$ respectively quantify the error in the average and standard deviation of the grain size. $n^{(i)}$ is the number of microstructural instantiations that belong to the bin $i . \overline{E S D}_{j}^{(i)}$ and $S_{j}^{s d}(i)$ represent the average and standard deviation of the diameter of the equivalent 
spherical grain in the $j$ th synthetic microstructure belonging to bin $i . \overline{E S D}$ and $S^{s d}$, on the other hand, correspond to values from stereological analysis of EBSD maps in table 1. Figure 2 shows the error in the average and standard deviation as a function of the number of grains in the synthetic microstructure. The error generally decreases rapidly with increasing number of grains. Less than $2 \%$ error is seen for both parameters for microstructures containing more than 480 grains.

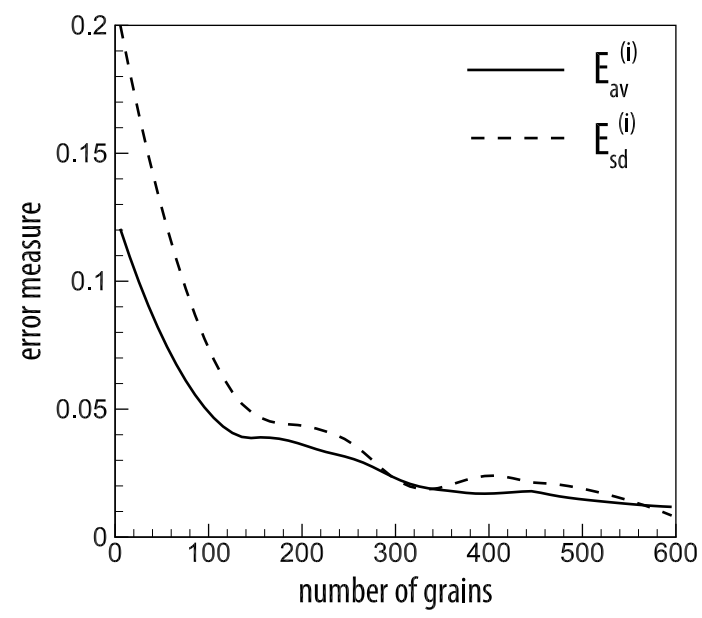

Fig. 2. Error in the the average and standard deviation of 3D grain size for the rolled-annealed (RA) microstructures.

To assess the fidelity of crystallographic distribution representation in the synthetic microstructure, the misorientation distribution is examined. The error in the misorientation distribution is evaluated by the Kolmogorov-Smirnov (KS) test (Massey, 1951). It is a distribution-free test of goodness of fit, which quantifies the maximum difference between two cumulative distribution functions (CDFs). Figure 3 shows that the KS statistic generally decreases rapidly with increase in the number of grains in the microstructure. In other words, the misorientation CDF of the synthetic microstructure compares better to the one obtained from EBSD maps with increasing the number of grains.

From the results in figures 2 and 3 for the RA microstructure, a statistically equivalent microstructure of dimensions $960 \times 960 \times 960 \mu \mathrm{m}^{3}$ containing 529 grains is chosen for crystal plasticity FE simulations. Figure 4(a) shows the contour plot of the $\langle c\rangle$-axis misorientation of a microstructure containing 529 grains. The pole figures, misorientation and grain size distribution of the 529-grain simulated microstructure are compared with those obtained from EBSD maps in figures 4(b-d). Very good agreement is seen between the simulated and the experimental probability density functions. 


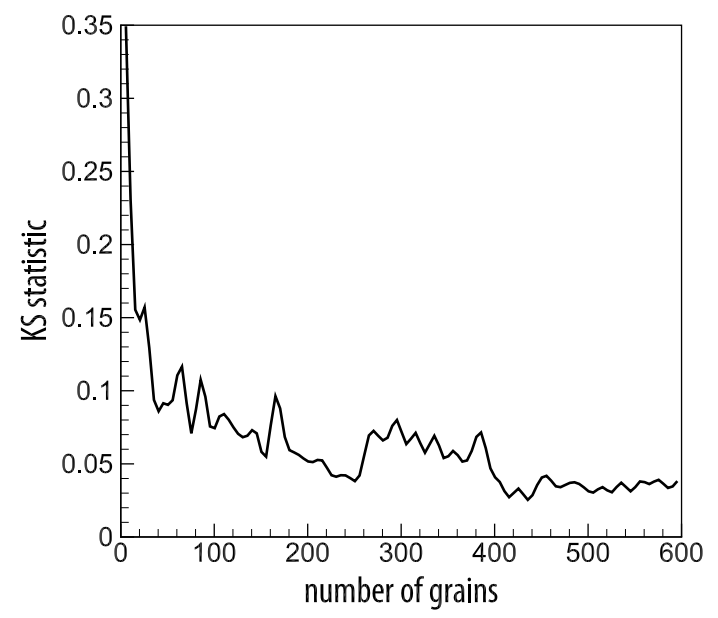

Fig. 3. Kolmogorov-Smirnov test for the misorientation distribution in the rolled-annealed (RA) microstructures.

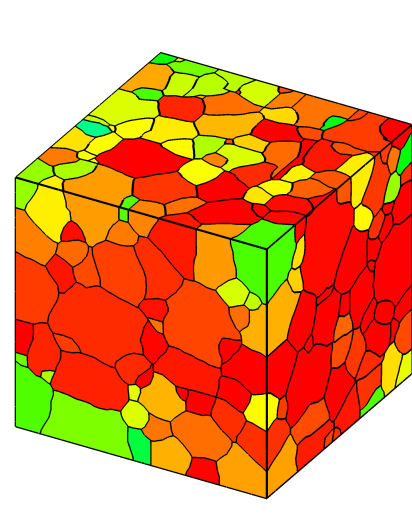

(a)

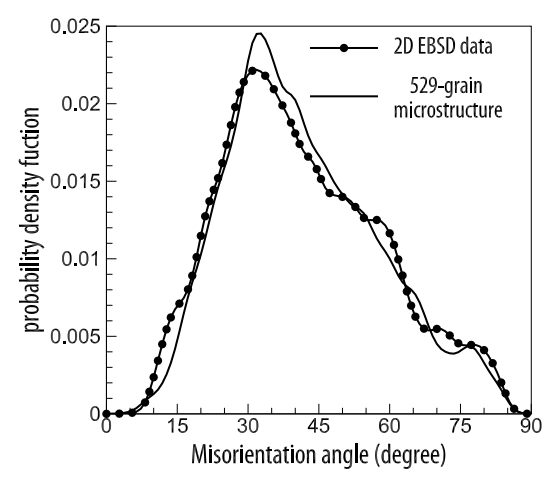

(C)

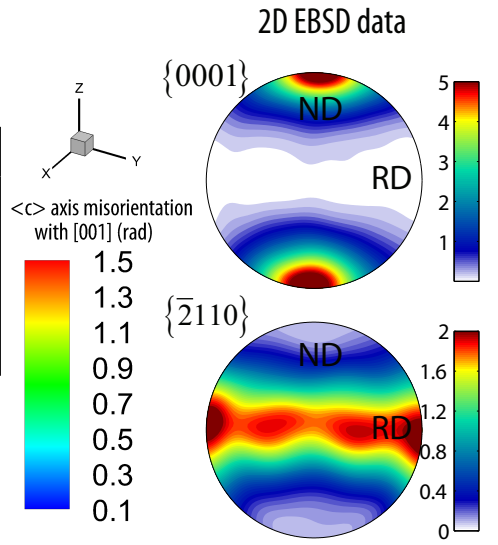

(b)

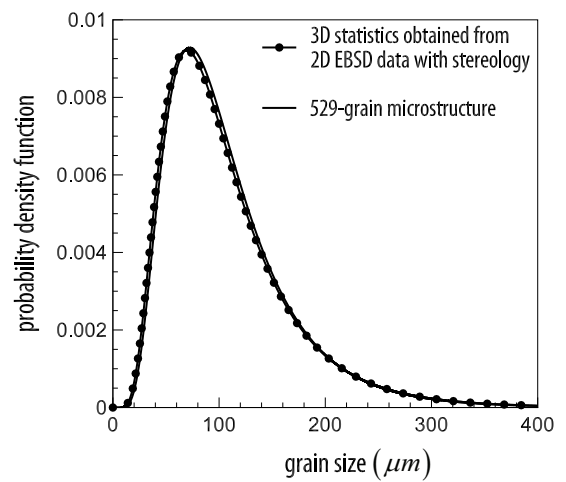

(d)

Fig. 4. (a) Contour plot of $\langle c\rangle$-axis misorientation for the 529-grain statistically equivalent RA virtual microstructure; Comparison of (b) orientation distribution, (c) misorientation distribution and (d) grain size distribution of the simulated microstructure with those from the EBSD data. 
With an analogous reconstruction process and convergence study, a statistically equivalent RVE of dimensions $300 \times 300 \times 300 \mu m^{3}$ containing 515 grains is simulated for the AR sample. Figure 5)(a) shows the $\langle c>$-axis misorientation contour plot, while pole figures, misorientation and grain size distribution of the simulated microstructure are compared with EBSD based results in figures 5 (b-d).

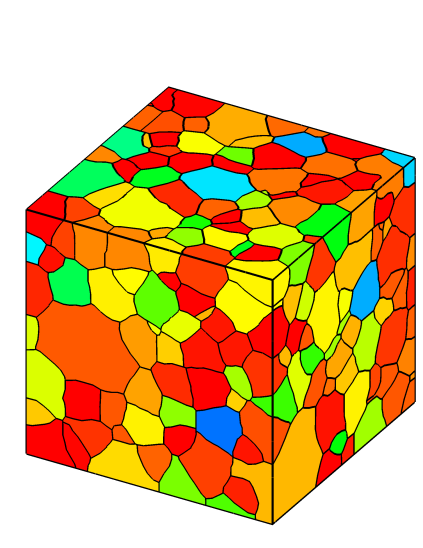

(a)

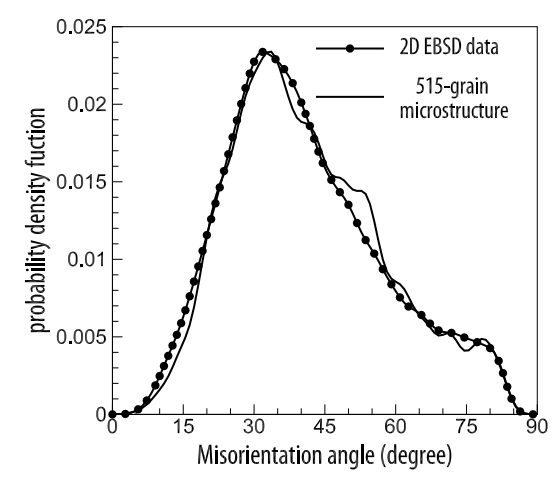

(C)

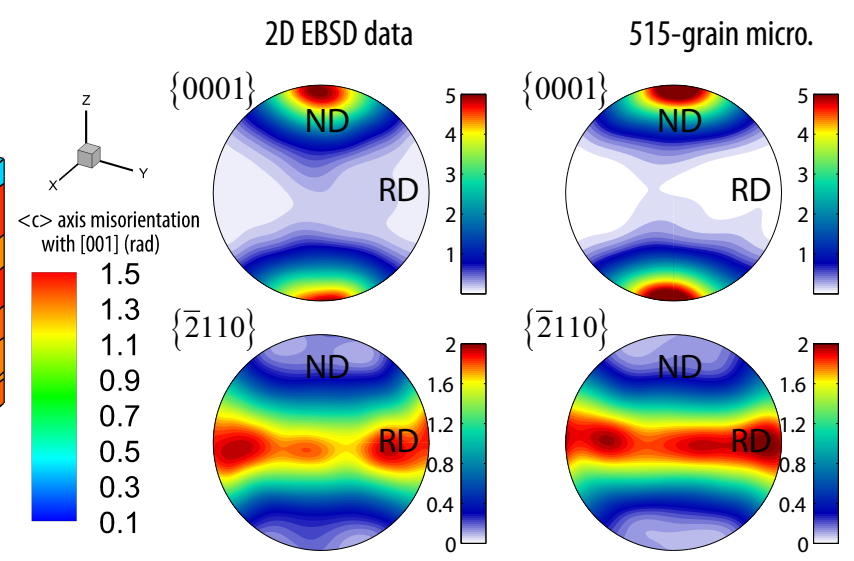

(b)

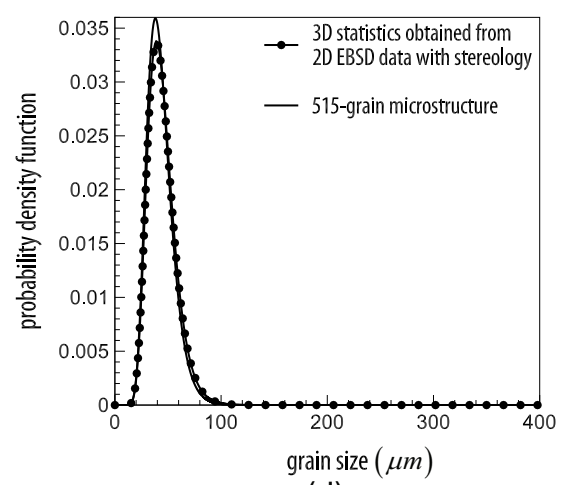

(d)

Fig. 5. (a) Contour plot of $\langle c\rangle$-axis misorientation for the 515-grain statistically equivalent AR virtual microstructure; Comparison of (b) orientation distribution, (c) misorientation distribution and (d) grain size distribution of the simulated microstructure with those from the EBSD data.

\subsection{Mesh Generation and Convergence Study}

The 3D simulated statistically equivalent microstructures, developed in the previous section, are represented as voxelized volumes with each voxel in a grain identified by a common set of 3 Euler angles. For crystal plasticity FE analysis, these voxelized volumes should be transformed into domains with smooth grain boundaries that can be discretized into a finite element mesh. For discretization of the computational domain, the voxelized synthetic microstructures are input into the Simmetrix ${ }^{\circledR}$ software (Simulation Modeling Suite, 
2015). For enhancing mesh quality, this software applies some basic filters to remove the small features and smoothen the grain boundaries. Stabilized four-node tetrahedral or TET4 elements, with linear interpolation functions for displacements, discretize the polycrystalline domains. The aspect ratio of elements in the mesh is kept below 70 and less than $0.01 \%$ of elements have an aspect ratio higher than 40 .

It is necessary to conduct a mesh convergence study with respect to both macroscopic and microscopic variables, prior to conducting major CPFE simulations (Diard et al., 2005). For this study, a CPFE analysis of the virtual RA microstructure in figure 4(a) is conducted under constant strain-rate $\dot{\epsilon}=1.1 \times 10^{3} \mathrm{~s}^{-1}$ along the [100] or x-direction. Two mesh densities with 536090 and 754916 elements are considered for comparison. Figure 6(a) shows the comparison of the macroscopic volume-averaged stress-strain response, while figure 6(b) compares the distribution of microscopic von Mises stress along an X-directed line passing through the centroid of the microstructure. The mesh convergence study concludes that the 536090-element mesh provides sufficient resolution for the CPFE simulations. A similar study for the AR microstructure converges to a 517023-element mesh to provide adequate accuracy.

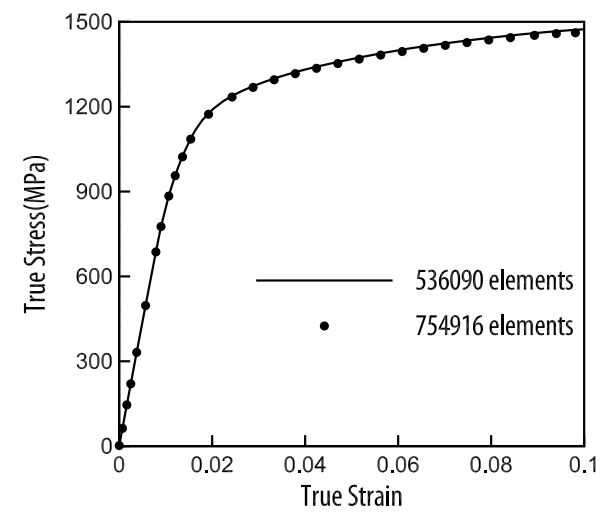

(a)

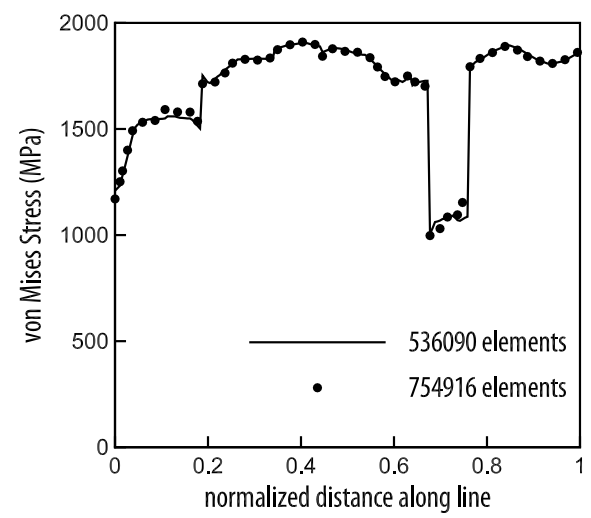

(b)

Fig. 6. Mesh convergence study for the RA microstructure by comparing results of two mesh densities: (a) volume-averaged loading direction stress-strain response and (b) von Mises stress at $2 \%$ strain along an X-directed line.

\section{Mechanical Testing of Polycrystalline Ti-7Al Specimen}

The unified flow rule (UL) and phenomenological (PL) crystal plasticity models are calibrated and validated using experiments that are conducted at Army 
Research Laboratories in Aberdeen. Room temperature, uniaxial compression experiments are conducted to determine the mechanical behavior of both the as-rolled (AR) and rolled-annealed (RA) materials. The tests are performed at both, quasi-static $\left(10^{-3} s^{-1}\right)$ and dynamic strain rates $\left(1000-4000 s^{-1}\right)$ to investigate strain-rate sensitivity. Given the texture in both materials, it was necessary to run the experiments with loading along the three orthogonal directions (normal (ND), rolled (RD), and transverse (TD)) to probe possible anisotropic behavior.

\subsection{Experimental Methods}

The quasi-static (QS) tests are conducted on a screw-driven Instron load frame under displacement control conditions. The specimens are machined from the bulk materials using a wire-EDM to dimensions of $3.5 \times 3.5 \times 7 \mathrm{~mm}$ (aspect ratio of 2). A compression subpress fixture ensures proper axial alignment during loading. To minimize the frictional effects, the ends of the specimens are lubricated. The displacement is measured using a stereoscopic digital image correlation (DIC) system consisting of two $2.3 \mathrm{MP}$ cameras. The choice of a two-camera system is made to eliminate the effect of out of plane motion on the strain measurements. DIC speckle pattern is applied to the surface of the specimen using a fine airbrush. VicSnap and Vic3D are used to acquire the images and perform the correlation (subset 29, step 10). Uniaxial strain is calculated using a digital extensometer. None of the specimens failed during testing. Unloading is initiated either after sufficient data is obtained or the specimen begins to deform in a non-uniform manner upon which the data becomes invalid.

The dynamic (DY) strain-rate tests are conducted on a compression Kolsky (Split-Hopkinson) bar, a technique originally suggested in Hopkinson (1914) and subsequently modified in (Hopkinson, 1949). It is currently the most popular technique for testing of high strain-rate $\left(10^{3}-10^{4} s^{-1}\right)$ behavior of materials. The Kolsky bar consists of two $9.5 \mathrm{~mm}$ maraging steel bars, referred to as the input and output bars, with the specimen sandwiched between them. A gas gun accelerates a projectile, which strikes the input bar, creating a compressive stress pulse that travels down the input bar and loads the specimen (Huskins et al., 2010). The foil strain gages, located on the input and output bars, record the reflected and transmitted stress pulses respectively. These data are used to calculate the stress and strain rate history of the specimen once it has reached stress equilibrium. The strain-rate history is integrated over time to obtain the strain history, which is correlated with the stress history to form stress-strain curves. A complete description of the Kolsky bar 
experimental technique is provided in (Chen and Song, 2011). The specimens are machined from the bulk materials to dimensions of $3.5 \times 3.5 \times 2.5 \mathrm{~mm}$ (aspect ratio of 0.7 ). The smaller aspect ratio reduced the inertial effects during testing and is inversely related to the achievable strain rate. The specimen ends were polished and lubricated to limit the effects of friction.

\subsection{Experimental Results}

Figure 7 shows the representative compressive true stress-strain curves for the rolled-annealed (RA) and as-rolled (AR) materials. Valid measurement of stresses and strain-rates using the Kolsky bar experiments requires stress equilibrium within the specimen. Since this condition may not be satisfied at the early stages of deformation, corresponding to very small strains, the calculated strain-rates and stresses are not representative of the mechanical response of the material under uniaxial loading conditions. In this work, the strains at which stress equilibrium is reached is calculated to be $\sim 2-3 \%$. The Youngs modulus and the $0.2 \%$ offset yield stress are therefore obtained from the QS data only. The transverse and rolled directions for the RA material have similar response. However, the normal direction shows increased strength and strain hardening. The $0.2 \%$ yield strengths for the RD, TD, and ND are $613 M P a, 620 M P a$, and $909 M P a$ respectively, which corresponds to an increase in strength of over $30 \%$ in the ND. The $5 \%$ flow stress shows increased strength of $265 \mathrm{MPa}, 302 \mathrm{MPa}$, and $209 \mathrm{MPa}$ for the RD, TD, and ND respectively. The direction-averaged Youngs modulus is $122 \mathrm{GPa}$.

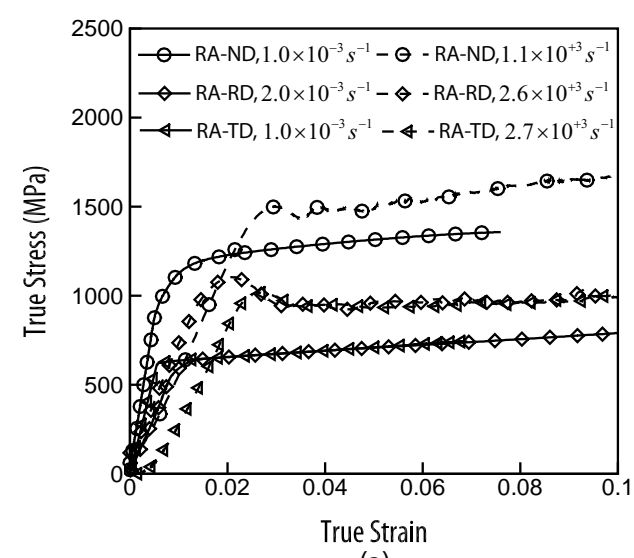

(a)

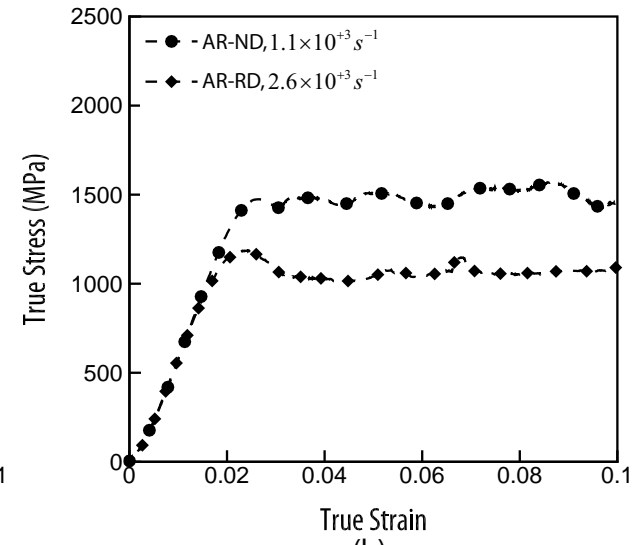

(b)

Fig. 7. Representative compression true stress-strain curve for the Ti-7Al alloy tested at QS and DY strain rates with: (a) RA and (b) AR material microstructures. 


\section{Calibration and Validation of the Image-Based Crystal Plasticity FE Model with the Unified Flow Rule}

Image-based CPFE models need robust constitutive models for predicting the material response. Both the unified flow rule (UL) and phenomenological flow rule (PL) discussed in part I (Shahba and Ghosh, 2016), are incorporated for CPFE analyses. The constitutive models are calibrated and validated using the results of the quasi-static and dynamic experiments discussed in section 3 , Results from eight experiments are used for calibration of the crystal plasticity constitutive model parameters and also validation of the CPFE model. The parameters and conditions for these tests are tabulated in tables 2 and 3 , The different variables in the table corresponds to:

(i) the microstructure variations due to variation in the processing conditions: as-rolled (AR) or rolled-annealed (RA),

(ii) the applied strain-rate corresponding to quasi-static (QS) or dynamic (DY) loading classification, and

(iii) the loading direction that may be normal (ND), rolling (RD) or transverse (TD) direction.

The ND, RD and TD directions correspond to the global [100], [010] and [001] directions respectively in the simulations. Four of the eight experiments given in table 2 are used for calibration of constitutive parameters, while the other four in table 3 are used for CPFE model validation. Both the calibration and validation experiments incorporate a diverse set of conditions.

Table 2

\begin{tabular}{|c|c|c|c|}
\hline Microstructure & Strain-rate $\left(s^{-1}\right)$ & Classification & Loading direction \\
\hline RA & $1.0 \times 10^{-3}$ & QS & ND \\
RA & $1.1 \times 10^{+3}$ & DY & ND \\
RA & $2.0 \times 10^{-3}$ & QS & RD \\
RA & $2.6 \times 10^{+3}$ & DY & RD \\
\hline
\end{tabular}

Specifics of calibration experiments.

\begin{tabular}{|c|c|c|c|}
\hline Microstructure & Strain-rate $\left(s^{-1}\right)$ & Classification & Loading direction \\
\hline RA & $1.0 \times 10^{-3}$ & QS & TD \\
RA & $2.7 \times 10^{+3}$ & DY & TD \\
AR & $1.1 \times 10^{+3}$ & DY & ND \\
AR & $2.6 \times 10^{+3}$ & DY & RD \\
\hline
\end{tabular}

Table 3

Specifics of validation experiments. 


\subsection{Calibration of Constitutive Parameters}

The constitutive equations for the unified flow rule (UL) and phenomenological (PL) crystal plasticity models are given in details in section 2 of part I of this paper (Shahba and Ghosh, 2016). Constitutive parameters to be calibrated include the anisotropic elastic constants and slip system-dependent crystal plasticity parameters. The $\alpha$ titanium alloys with a $h c p$ lattice-parameter ratio of $c / a=1.59$ shows a transversely isotropic elastic response. The elastic stiffness coefficients are expressed in a material coordinate system that is defined by the orthonormal basis $\left(\mathbf{e}_{1}^{c}, \mathbf{e}_{2}^{c}, \mathbf{e}_{3}^{c}\right)$. Here, the directions 1, 2 and 3 correspond respectively to [1210], [1010] and [0001] directions of the hcp crystal lattice, as depicted in figure 2 of part I (Shahba and Ghosh, 2016). The anisotropic elasticity tensor in this coordinate system is expressed in the Voigt notation as $\left[C_{I J}^{e}\right], I, J=1 \cdots 6$. For transversely isotropic elastic behavior, there are only 5 independent elastic constants, viz. $C_{11}=C_{22}, C_{12}, C_{13}=C_{23}, C_{33}, C_{55}=$ $C_{66}$ and $C_{44}=\left(C_{11}-C_{12}\right) / 2$. The elastic constants are measured using resonant ultrasound spectroscopy experiments on Ti-7 single crystal samples at room temperature in (Shade, 2015) and tabulated in Table 4.

Experimental measurements of elastic constants of $\alpha$-Ti have shown a near linear dependence on temperature (Ogi et al., 2004). Stiffness components decrease almost linearly with increasing temperature but with different slopes. Experimental results in (Ogi et al., 2004) are used to obtain the thermal gradients of the stiffness components. The linear slopes of elastic coefficients with temperature are given in Table 4 .

\begin{tabular}{|c|c|c|c|c|c|c|}
\hline Stiffness Parameters & unit & $I J=11$ & $I J=12$ & $I J=13$ & $I J=33$ & $I J=55$ \\
\hline$C_{I J}$ & $\mathrm{GPa}$ & 164.7 & 82.5 & 61.8 & 175.2 & 48.5 \\
$-\frac{\partial C_{I J}}{\partial T}$ & $\mathrm{MPa} / \mathrm{K}$ & 48 & 8.9 & 21 & 21 & 21.9 \\
\hline
\end{tabular}

Table 4

Experimentally measured elastic stiffness components at room temperature in (Shade, 2015), and their slopes with temperature (Ogi et al., 2004).

The crystal plasticity parameters for the (UL) and (PL) models are calibrated in an iterative manner. For the (PL) model, the parameters that have been calibrated for the Ti-6Al alloy in (Hasija et al., 2003) are taken as the starting values since its chemical composition is relatively close to that of $\mathrm{Ti}-7 \mathrm{Al}$ alloy. From sensitivity analysis, it is revealed that $\dot{\gamma}_{0}^{\alpha}, s_{0 *}^{\alpha}$ and $m$ are the parameters that control the onset of plasticity i.e. yield point. Also the parameters $h_{r e f}^{\alpha}, \tilde{s}^{\alpha}, r^{\alpha}$ and $n^{\alpha}$ are the ones controlling the hardening rate. The exponent $m$ is found to be the primary parameter controlling rate-sensitivity. The calibration process is designed to use quasi-static tests at room temperature to calibrate parameters that control the onset of plasticity as well as hardening. 
Dynamic tests are utilized to calibrate the rate-controlling parameter $m$ that affects the yield stress, as well.

Using the four calibration tests categorized in table 2, parameters in the (PL) model are calibrated. The volume-averaged stress-strain plots, comparing simulated results with experiments are shown in figure 8. The hatched area in figure $8 \mathrm{a}$ corresponds to a period at which stress equilibrium is not necessarily reached in the sample during high-rate experiments. Therefore, the experimental stress-strain response is not reliable during this period. The calibrated parameters for the (PL) model are given in table 5. The response to loading in the ND direction is stiffer than in the RD direction. This is the effect of the rolling process, which aligns the $\langle c\rangle$ - axis of grains along the ND direction. Therefore, loading along the ND direction will favor activation of $\langle c+a\rangle-$ pyramidal slip systems whose critical resolved shear stress is $2 \sim 3$ times larger than the $\langle a\rangle$ - basal or prismatic slip systems (Li et al., 2013).

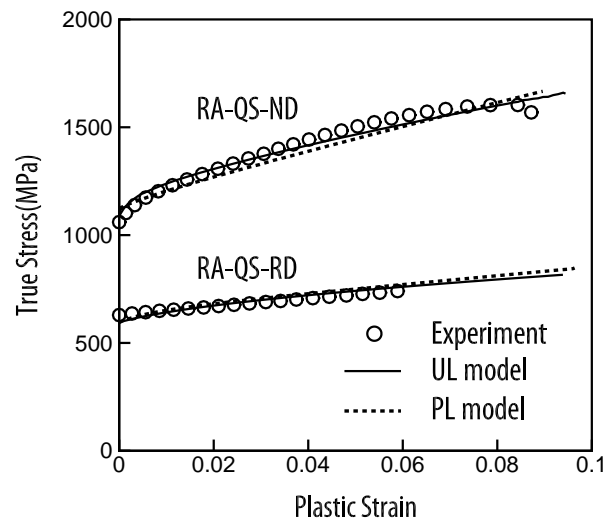

(a)

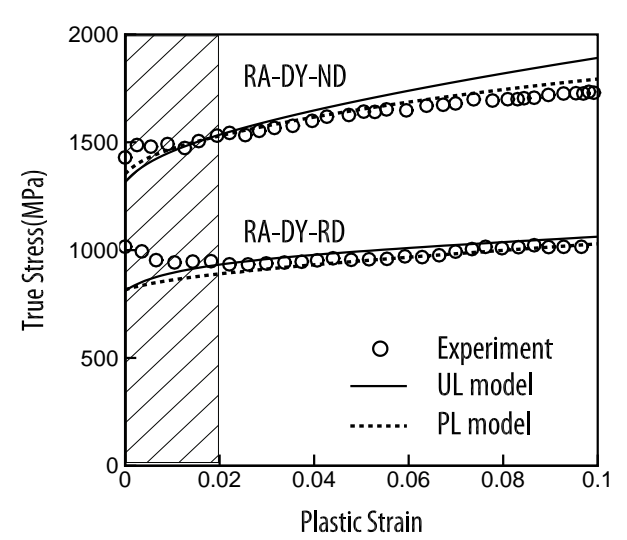

(b)

Fig. 8. Stress-strain plots used in the calibration of the (PL) and (UL) model parameters using: (a) quasi-static (QS) and (b) dynamic (DY) experiments. The hatched region corresponds to the range where stress equilibrium is not attained in the dynamic experiments.

It is also important to account for the effect of temperature increase on plastic deformation in high strain-rate simulations due to adiabatic heating. Increasing temperature boosts the rate of successful thermal activation attempts and consequently plastic flow is enhanced. In other words, the resistance to plastic flow reduces with rising temperature. In the (PL) model, this is phenomenologically accounted for by scaling the slip system resistances with temperature as: $s_{*}^{\alpha}=s_{r e f *}^{\alpha}\left(T / T_{r e f}\right)^{p}$, where $s_{r e f *}^{\alpha}$ is the slip system resistance at reference temperature $T_{r e f}$ and $p$ is a fitting parameter (Clayton, 2005). In the present work, $T_{r e f}$ is set to room temperature, for which $s_{r e f *}^{\alpha}$ will correspond to $s_{0 *}^{\alpha}$. The $p$ 


\begin{tabular}{|c|c|c|c|c|}
\hline $\begin{array}{c}\text { Parameters } \\
\text { (units) }\end{array}$ & $\langle a\rangle$ basal & $\langle a>$ prismatic & $\langle a\rangle$ pyramidal & $\langle c+a\rangle$ pyramidal \\
\hline$s_{0 *}^{\alpha}(\mathrm{MPa})$ & 230 & 205 & 500 & 610 \\
$\dot{\gamma}_{0}^{\alpha}\left(s^{-1}\right)$ & 0.003 & 0.003 & 0.003 & 0.003 \\
$h_{r e f}^{\alpha}(\mathrm{MPa})$ & 250 & 250 & 1200 & 2000 \\
$m$ & 0.019 & 0.019 & 0.019 & 0.019 \\
$r^{\alpha}$ & 0.02 & 0.02 & 0.02 & 0.02 \\
$n^{\alpha}$ & 0.3 & 0.3 & 0.3 & 0.3 \\
$\tilde{s}^{\alpha}(\mathrm{MPa})$ & 1600 & 1600 & 1600 & 1800 \\
\hline
\end{tabular}

Table 5

Calibrated parameters of the (PL) constitutive model for different slip systems.

exponent is set to -1 using the experimental results in (Williams et al., 2002) on $\alpha$-Ti alloys. They have measured the variation of yield stress and critical resolved shear stresses for different slip systems at a wide range of temperatures. The results in (Williams et al., 2002) are laterally shifted such that yield stress at room temperature matches the one using the calibrated parameters in table 5. Figure 9 shows the variation of $0.2 \%$ yield stress with temperature, for single crystal Ti-7Al that is oriented for activation of $\langle a\rangle$-basal and $\langle c+a\rangle$-pyramidal slip systems. The results of $\langle a\rangle$-prism slip are not shown since it is very close to the response of $\langle a\rangle$-basal slip.

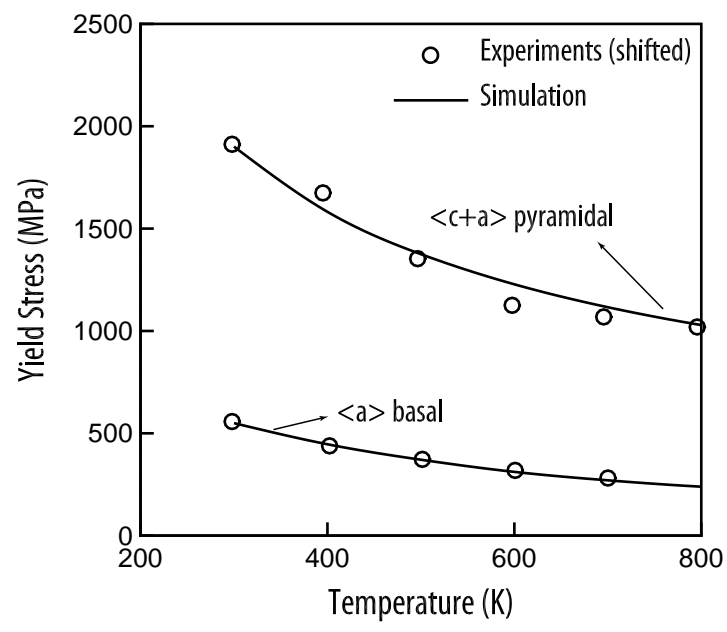

Fig. 9. Variation of yield stress with temperature for single crystals oriented for activation of different slip systems.

The crystal plasticity parameters for the unified flow rule (UL) crystal plasticity model are calibrated in the same way as for the (PL) model. Exper- 
imental observations (Akhtar and Teghtsoonian, 1975; Tanaka and Conrad, 1972) suggest that the effective activation energy is generally temperaturedependent. Hence the effective activation energy is expressed in terms of temperature as:

$$
Q_{s l i p}^{\alpha}(T)=Q_{r e f}^{\alpha}+c_{Q}^{\alpha}\left(T / T_{r e f}-1\right)^{p_{Q}^{\alpha}}
$$

These parameters are calibrated using experimental data in (Williams et al., 2002). The calibrated parameters for the (UL) model are delineated in Table 6 . The corresponding comparison with experimental stress-strain plots are shown in figure 8 ,

\begin{tabular}{|c|c|c|c|c|}
\hline $\begin{array}{c}\text { Parameters } \\
\text { (units) }\end{array}$ & $\langle a\rangle$-basal & $\langle a\rangle$-prismatic & $\langle a\rangle$-pyramidal & $\langle c+a\rangle$-pyramidal \\
\hline$Q_{\text {ref }}^{\alpha}(\mathrm{J})$ & $2.1 \times 10^{-19}$ & $2.2 \times 10^{-19}$ & $3.0 \times 10^{-19}$ & $2.6 \times 10^{-19}$ \\
$l_{\text {kink }}^{\alpha}\left(b^{\alpha}\right)$ & 20 & 20 & 20 & 20 \\
$s_{0 *}^{\alpha}(\mathrm{MPa})$ & 5.0 & 5.0 & 5.0 & 5.0 \\
$c_{a t h}^{\alpha}$ & 0.8 & 0.62 & 0.7 & 0.5 \\
$c_{a c t}^{\alpha}$ & 0.7 & 0.7 & 0.1 & 0.04 \\
$c_{l}^{\alpha}$ & 8.0 & 8.0 & 8.0 & 8.0 \\
$c_{m u l t i}^{\alpha}\left(\mu m^{-1}\right)$ & 150 & 230 & 500 & 500 \\
$c_{a n n i h}^{\alpha}$ & 10 & 10 & 10 & 10 \\
$c_{Q}^{\alpha}(\mathrm{J})$ & $2.3 \times 10^{-20}$ & $3.7 \times 10^{-20}$ & $1.8 \times 10^{-20}$ & $0.9 \times 10^{-20}$ \\
$p_{Q}^{\alpha}$ & 1.6 & 1.6 & 1.6 & 1.6 \\
\hline
\end{tabular}

Table 6

Calibrated parameters of the (UL) model for different slip systems.

\subsection{Validation of the CPFE Model with Experimental Results}

Validation of both the (UL) and (PL) models with respect to the fidelity of calibrated parameters is achieved by simulating the four experiments described in table 3, The image-based CPFE models for the AR and RA materials, developed in section 2, are used for these simulations and boundary conditions are applied commensurate with the experimental loading conditions. Results of the simulations are compared with corresponding experiments in figure 10. Generally a good agreement is observed between the models and experiments for the range of strain-rates considered. Even though the parameters are calibrated using the RA materials, the models predict the deformation of AR microstructure very well. This concludes that the grain size-dependence mechanisms in the model, i.e. the GND hardening and Hall-Petch type effects are properly represented, since the primary difference between the AR and RA 
microstructures is the average grain size. Uniqueness of the calibrated parameters is always an issue when limited experimental results are available, as in this problem. The combination of sensitivity analysis and least-squares minimization routine, however yields the best possible combination of parameters to reproduce the experimentally observed response.

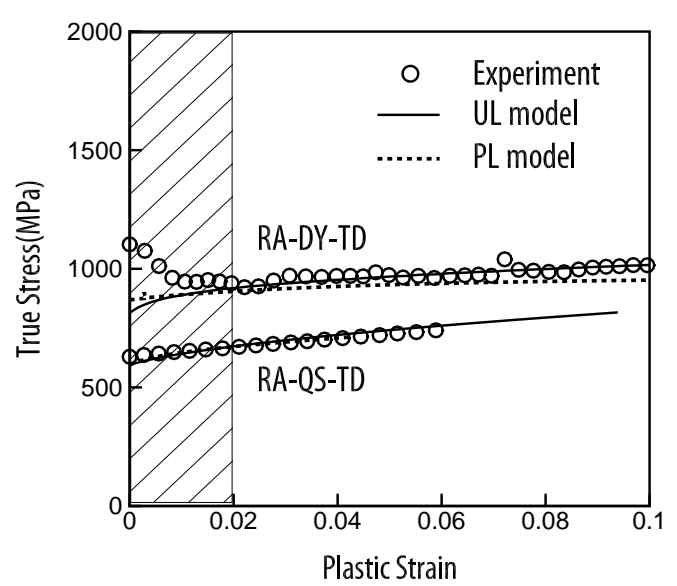

(a)

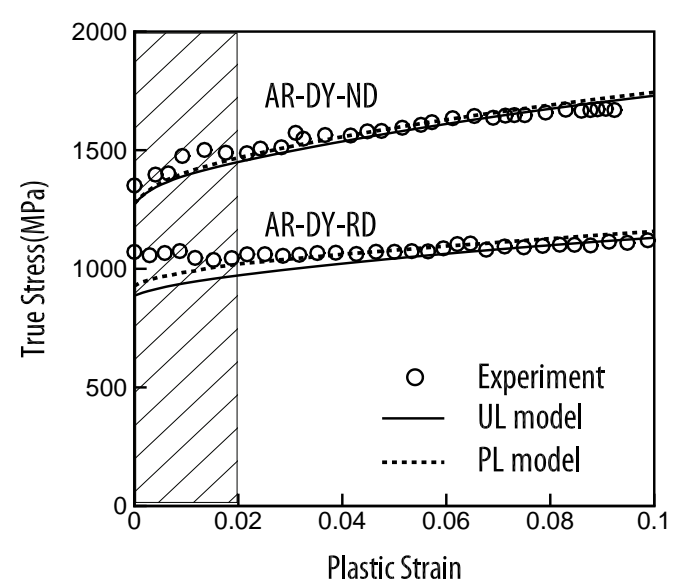

(b)

Fig. 10. Validation of (UL) and (PL) models for the quasi-static (QS) and dynamic (DY) experiments on (a) RA and (b) AR material microstructures.

\subsection{Validation of temperature-dependence of the flow stress}

Temperature changes affect both the elastic and plastic responses of $\mathrm{Ti}$ alloys as discussed in part I of this paper (Shahba and Ghosh, 2016). With increasing temperature, elastic stiffnesses decrease in value. This affects the slip-driven plasticity through a reduction in the shear modulus dependent slip system strength represented by the athermal stress (Ashby, 1970). Increasing temperature also promotes plasticity by boosting up the rate of successful thermal activation attempts. The effects of temperature on plastic deformation by the (UL) model is studied here under isothermal conditions.

Compression tests are simulated for the AR microstructure along ND and $\mathrm{RD}$ at a strain-rate of $10^{-3} s^{-1}$ under isothermal conditions, but at different initial temperatures. The volume-averaged stress-strain responses in the loading direction are plotted in figure 11a, With temperature increase, the yield stress decreases significantly but the reduction of elastic stiffness is negligible. Figure $11 \mathrm{~b}$ shows the variation of the yield stress with temperature in the temperature range of $300 K$ to $700 K$. It is observed that the yield stress decreases almost linearly with temperature, consistent with the experimental 
results in (Khan et al., 2004) performed on Ti-6Al-4V. The yield stress for the AR material in the ND direction decreases more rapidly in comparison with the RD direction. This is due to the crystallographic orientation of the grains and the dissimilar variation of critical resolved shear stress (CRSS) with temperature for different slip systems. Crystallographic orientations of grains in the AR material are such that the $\langle a\rangle$-basal and prism slip systems are the favorable modes of slip when the sample is loaded along the RD. On the other hand, $\langle c+a\rangle$-pyramidal slip is the favorable one for loading along the ND. As shown in figure 9, the CRSS for the $\langle c+a\rangle$-pyramidal slip system reduces at a faster rate with temperature, compared to that for the $\langle a\rangle$-basal and prism slip systems. This explains why the yield stress for the AR-ND simulations decreases more rapidly with temperature in figure $11 \mathrm{~b}$.

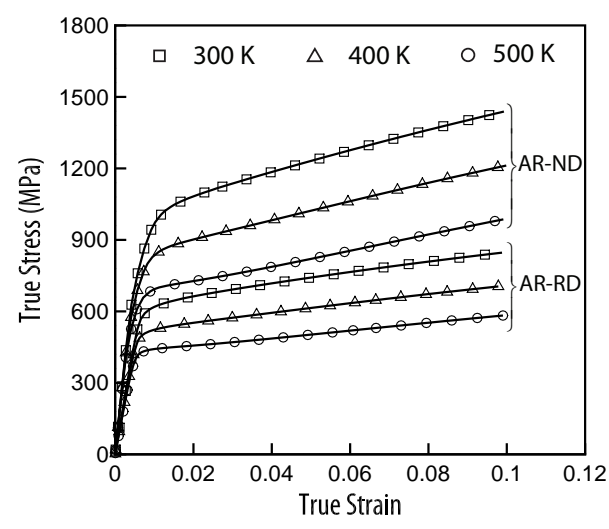

(a)

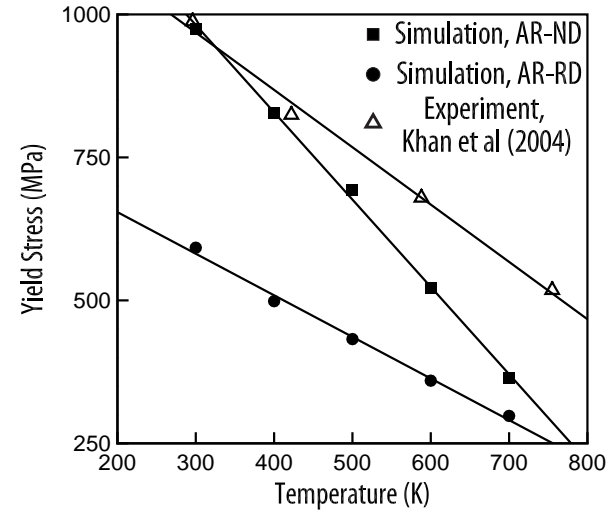

(b)

Fig. 11. CPFE simulation of the AR microstructure at different initial temperatures, subject to strain-rate of $10^{-3} s^{-1}$ : (a) loading direction stress-strain response, (b) variation of yield stress with temperature.

\section{Image-based CPFE Simulations with Adiabatic Heating}

This section explores the role of local adiabatic heating associated with high strain-rates, discussed in part I, on the deformation response of polycrystalline Ti-7Al alloys. The image-based CPFE models developed in section 2 are used for these simulations. The as-rolled (AR) material microstructural RVE is subjected to a compressive strain rate of $10^{4} \mathrm{~s}^{-1}$ along the normal direction (ND). Different case studies are considered for these simulations.

- Case I: Simulation in which local adiabatic heating is turned off, i.e. simulation is under isothermal conditions.

- Case II: Simulation in which local adiabatic heating is accounted for, but the reduction of elastic constants with temperature is neglected. 
- Case III: Simulation in which both local adiabatic heating and reduction of elastic constants with temperature are considered.

The macroscopic stress-strain response for the three cases are shown in figure 12. Comparing the responses, it is observed that the pre-yield portion of the stress-strain response is not affected much by the adiabatic heating, since the amount of plastic work and consequently the local temperature rise is very small in this stage of deformation. The effect of adiabatic heating becomes more evident with rise in the local temperature at higher strains. A lower strain hardening is seen for case III in comparison with case I for which the temperature evolution is suppressed. Moreover, based on the macroscopic response for cases II and III in figure 12, it is inferred that the effect of decrease in elastic constants with temperature becomes noticeable only at higher strains above 0.15 . Thus, the consideration of elastic softening is of secondary importance if material failure due to nucleation and evolution of microstructural defects starts at early stages of deformation. This is consistent with observations in (Osovski et al., 2013), where the effect of temperature on the failure of Ti alloys were investigated.

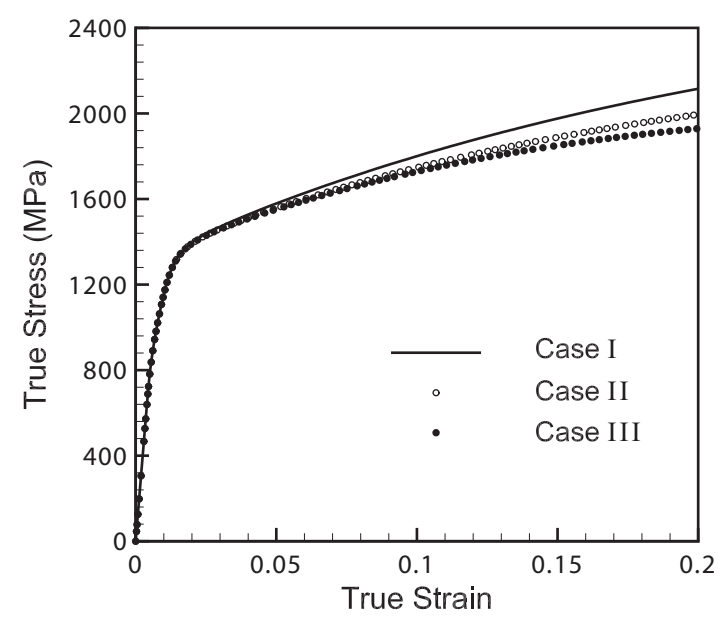

Fig. 12. Stress-strain response in the loading (ND) direction, for an applied compressive strain-rate of $10^{4} s^{-1}$ on the AR microstructural RVE.

Failure of Ti alloys at high strain-rates is attributed to formation of adiabatic shear bands (ASBs), which in turn is related to the emergence of hot spots due to adiabatic heating. The case III simulations are analyzed for local temperature evolution in each grain. A temperature increase $\Delta \bar{T}_{g}$ is calculated for each grain $g$, and its distribution over the entire microstructure at four different stages of deformation is plotted in figure 13a, The average temperature 
increase in the grain $g$ is evaluated as:

$$
\Delta \bar{T}_{g}=\frac{\sum_{i=1}^{N_{e}^{(g)}} \Omega_{i} \Delta \bar{T}_{i}}{\sum_{i=1}^{N_{e}^{(g)}} \Omega_{i}}
$$

where $N_{e}^{(g)}$ is the number of elements and $\Omega_{i}$ and $\Delta \bar{T}_{i}$ are respectively the volume and temperature increase in the $i$-th element of the grain $g$. The $\Delta \bar{T}_{g}$ distribution evolution indicates that not only the average temperature in individual grains, but also its standard deviation increases with deformation. A similar trend is observed in the distribution of the effective plastic strain in each grain, shown in figure $13 \mathrm{~b}$. The increase in heterogeneity of the plastic strain field implies that the micro-plasticity transitions from a nearly uniform state in the early stages of deformation to a non-uniform state in the later stages. This tendency of deformation towards a non-uniform distribution of the plastic strain implies the realization of severely plastically-deformed grains. Depending on the contiguity of these grains, large regions of localized deformation can be created. Figure 14 shows the formation of such a region over an $\mathrm{XZ}$ slice in the $3 \mathrm{D}$ microstructure. In this region, the temperature is also locally high due to significant dissipative plastic work, as observed in Figure 15. These regions can facilitate the nucleation of the adiabatic shear bands, initiating a failure process.

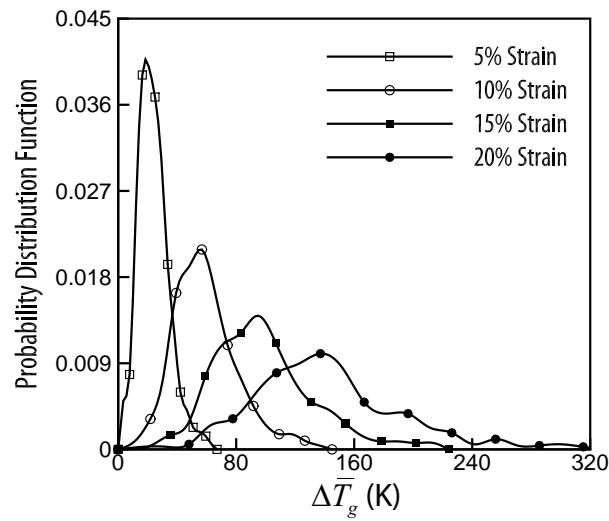

(a)

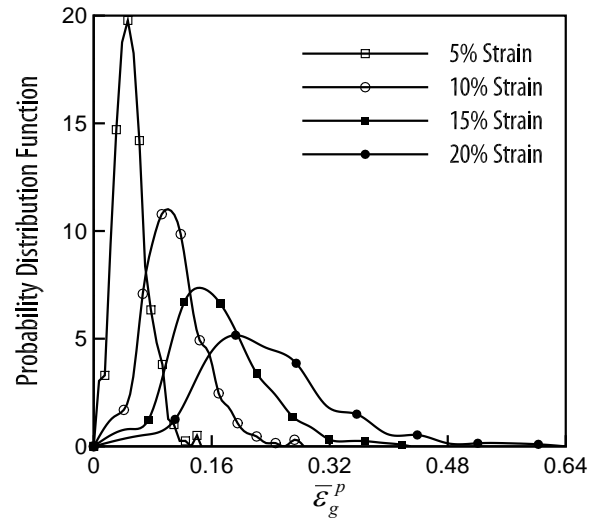

(b)

Fig. 13. Probability distribution functions of: (a) $\Delta \bar{T}_{g}$ and (b) $\bar{\epsilon}_{g}^{p}$ at different stages of deformation, in CPFE simulations of the AR microstructure at strain-rate of $10^{4} s^{-1}$. 

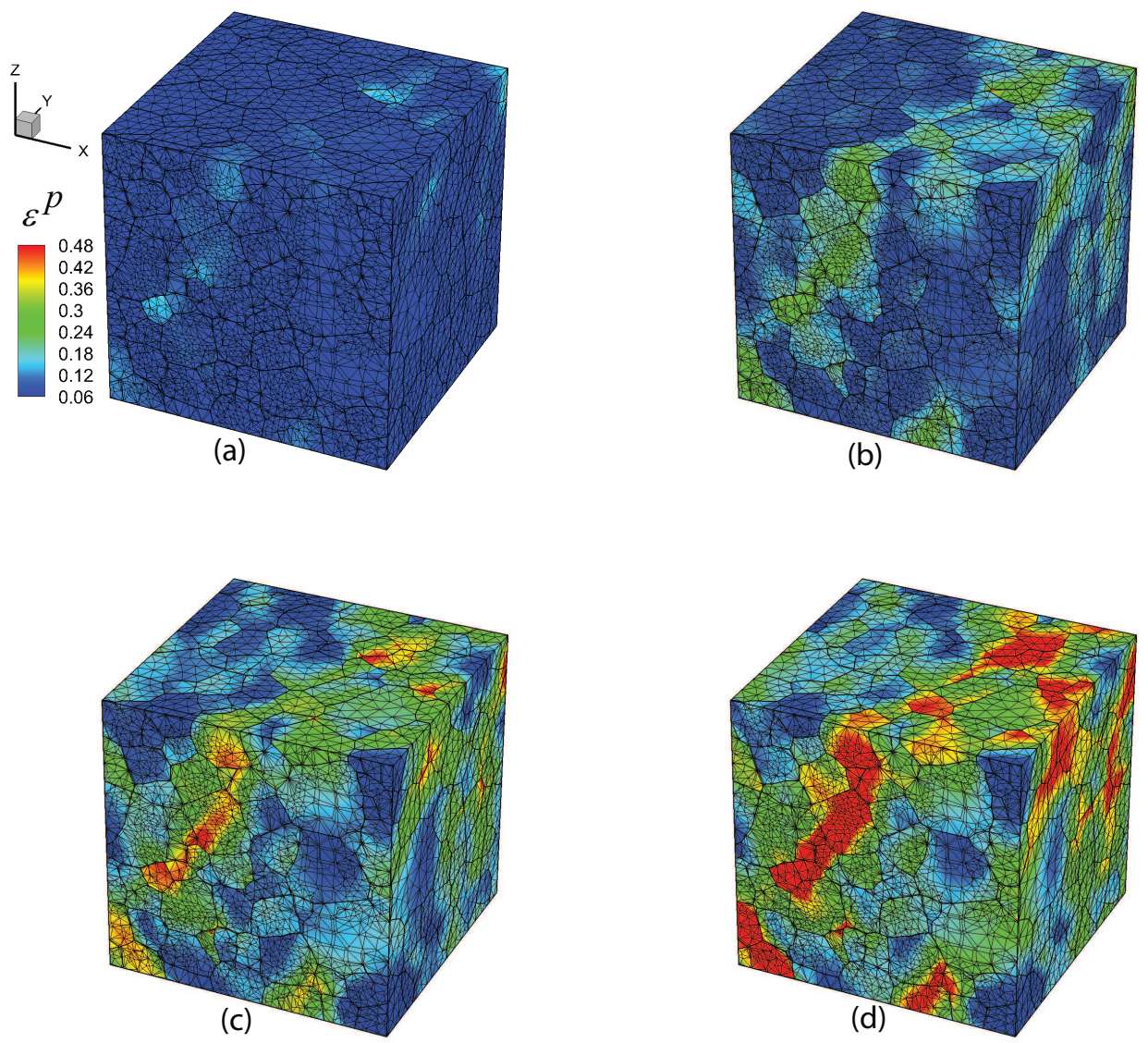

Fig. 14. Development of regions of plastic localization in the AR microstructure under a compressive strain-rate of $10^{4} \mathrm{~s}^{-1}$ along the ND direction, at: (a) 5\%, (b) $10 \%$, (c) $15 \%$ and (d) $20 \%$ strain. (The contours are shown in the undeformed configuration).

\subsection{An Anomaly in the Relation Between Temperature and Plastic Strain Hotspots}

Since the adiabatic heating is a consequence of plastic deformation, it may be reasonable to assume that the distributions in figure 13 are complementary. This implies that with increased plastic localization in certain grains, the temperature rises adiabatically and causes an increase in the standard deviation in those grains in figure 13a, However, a close analysis of the plastic strain and temperature contours in the microstructure reveals that the regions with a high plastic strain do not necessarily correspond to hot spots of the temperature field and vice versa. For example, the effective plastic strain contour plot in figure 16a shows that grain $A$ is highly plastically deformed. However the contour plot of temperature field in figure $16 \mathrm{~b}$ does not suggest an elevated temperature in this grain. On the other hand, grains $B, C$ and $D$ experience elevated thermal fields, although they are undergoing moderate plastic defor- 

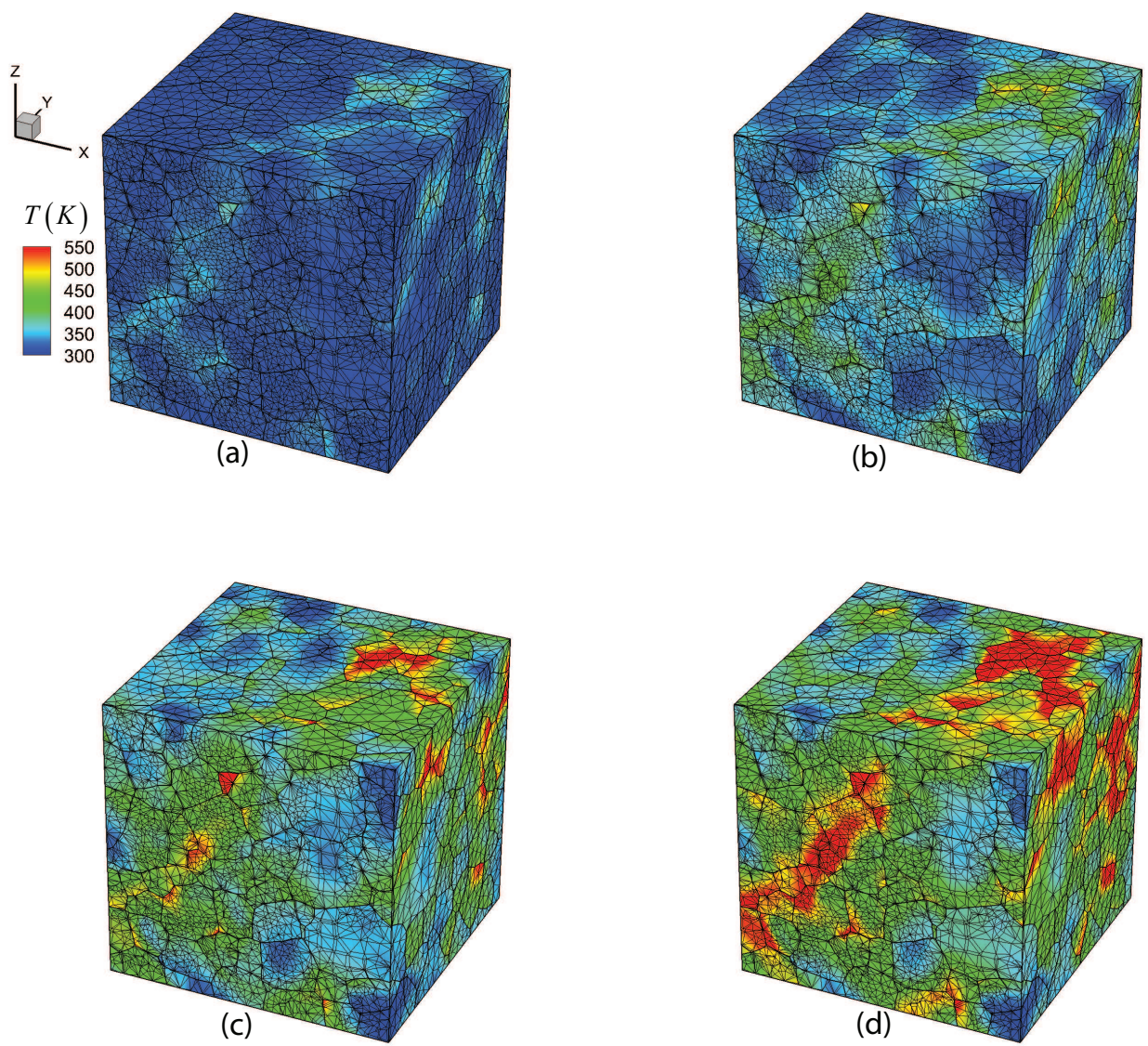

Fig. 15. Evolution of temperature in the AR microstructure under a compressive strain-rate of $10^{4} s^{-1}$ along the ND direction, at: (a) $5 \%$, (b) $10 \%$, (c) $15 \%$ and (d) $20 \%$ strain. (The contours are shown in the undeformed configuration)

mation. To comprehend this anomalous behavior, the plastic power density definition is recalled as:

$$
\dot{W}_{p}=\boldsymbol{\sigma}: \mathbf{d}^{p}
$$

where $\boldsymbol{\sigma}$ and $\mathbf{d}^{p}$ are respectively the Cauchy stress and plastic part of the rate of deformation tensor in the current configuration. $\dot{W}_{p}$ is dependent on the stress level and the rate of plastic deformation in the multiplicative form. Hence, a high value of plastic strain by itself does not constitute a high value of plastic work and consequently adiabatic heating. Temperature may increase more at a material point with high level of stress but low plastic strain, compared to another material point with higher plastic strain and lower stress. Hence the temperature trends in grains $A, B, C$ and $D$ are more congruent with the contour plot of the von Mises stress, as a measure of stress tensor, as shown in figure $16 \mathrm{c}$

To study this further, a bi-crystal model consisting of a hard and soft grain is 


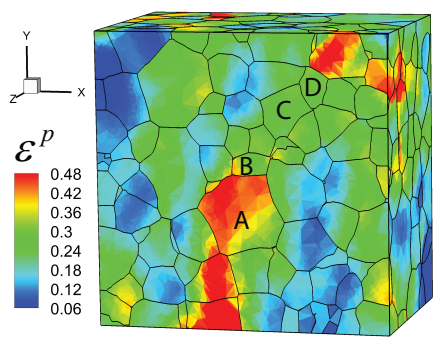

(a)

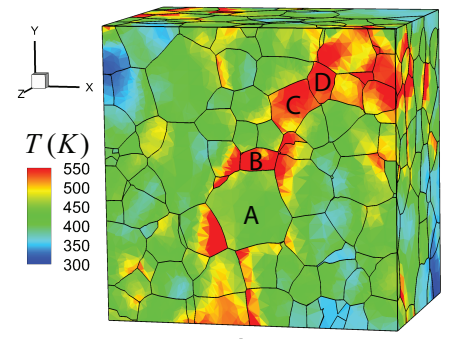

(b)

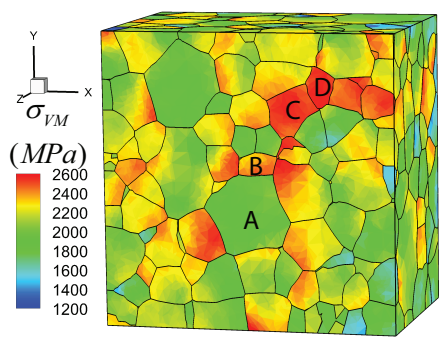

(c)

Fig. 16. Contour plots of: (a) effective plastic strain, (b) temperature and (c) von Mises stress at $20 \%$ strain, from CPFE simulations of the AR microstructure under a compressive strain-rate of $10^{4} s^{-1}$ along the ND direction.

generated as shown in figure 17a, The orientations of the soft and hard grains are identical to the ones shown in figures 8(a) and 8(c) respectively in part I of this paper (Shahba and Ghosh, 2016). The orientation induces a much higher level of plastic strain in the soft grain. Figure $17 \mathrm{~b}$ plots the macroscopic stressstrain response in the direction of loading, for this bi-crystal model with an applied compressive strain-rate of $10^{4} s^{-1}$ in the [001] direction. Three stages of deformation are selected as indicated in figure $17 \mathrm{~b}$. The evolution of relevant micro-mechanical variables are investigated at these stages along a line in the X-direction that passes through the centroid of the model. At stage I, the plastic flow has already started in the soft grain, whereas the hard grain has barely deformed plastically. As shown in figure 18a, the temperature in the soft grain is slightly higher than that in the hard grain. In the subsequent stages of deformation, the temperature in the hard grain increases significantly in comparison with the soft grain. Here again the rate of adiabatic heating is governed by the stress state. The temperature difference between the two grains grows larger with deformation as shown in figures $18 \mathrm{~b}$ and $18 \mathrm{c}$.

The results of the bi-crystal problem in figure 18 also provides some insight on the formation of adiabatic shear bands or ASBs at high strain-rates. Different criteria have been proposed for estimating the formation of adiabatic shear bands, based on thermo-mechanical variables, such as plastic shear strain in (Culver, 1973), temperature in (Medvanik et al., 2007) or stored energy of cold work in (Osovski et al., 2013; Rittel et al., 2006). The model developed in this paper with reference to figure 18 has the ability to comprehend the source of nucleation of such ASBs that will be studied more in future work. 


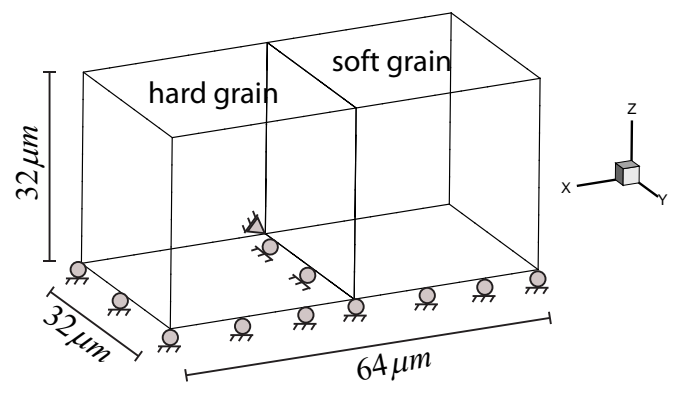

(a)

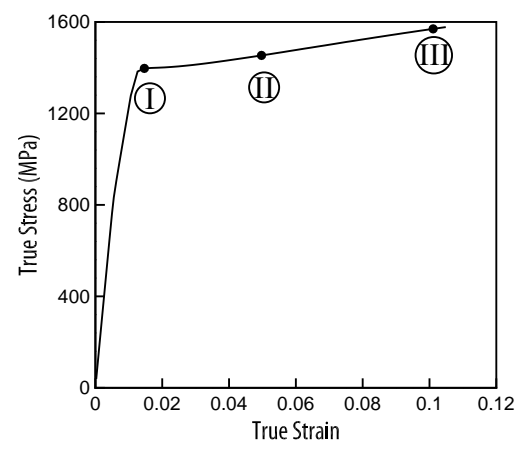

(b)

Fig. 17. (a) A bi-crystal model discretized into 5103 linear tetrahedral elements, and (b) macroscopic stress-strain response in the loading direction, for applied compressive strain-rate of $10^{4} s^{-1}$ along the [001] direction.

\section{Concluding remarks}

The second part of this paper develops an image-based crystal plasticity FE model, incorporating the unified flow rule (UL) and phenomenological flow rule (PL) based constitutive models, simulating deformation in the polycrystalline Ti-7Al alloy under low and high strain-rates. The image-based CPFE uses 3D statistically-equivalent virtual microstructures that are reconstructed by processing 2D EBSD surface data with statistical and stereological principles. The statistically equivalent virtual microstructures are constructed for the Ti-7Al alloy in as-rolled (AR) and rolled-annealed (RA) conditions. A set of quasi-static and dynamic tests are performed along different directions, viz. RD, TD and ND, to generate data for calibrating and validating the (UL) and (PL) constitutive models. Both the (UL) and (PL) models are calibrated from these experimental tests and also validated for accuracy. The effect of initial temperature is investigated for quasi-static isothermal simulations. The $0.2 \%$ yield stress decreases almost linearly with temperature in the temperature range of interest. The rate of decrease is observed to be higher along ND since the critical resolved shear stress for $\langle c+a\rangle$-slip system drops faster with temperature compared to that for the $\langle a\rangle$-slip systems.

Adiabatic simulations conducted with the image-based CPFE model incorporating the (UL) flow rule show that the effect of temperature on enhancing plasticity is more pronounced than its effect on reducing elastic constants. Analysis of high strain-rate simulations show the tendency of the microstructure towards localized plastic deformation with progress in material straining. Study on adiabatic heating reveals that the grains with severe plastic deformation do not necessarily show high localized temperatures as a result of conversion of plastic work into heat. It is likely that grains, which are not 

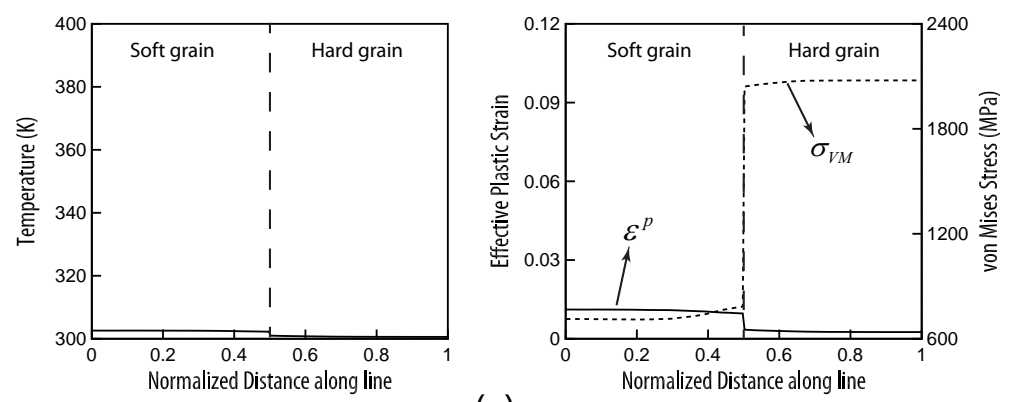

(a)
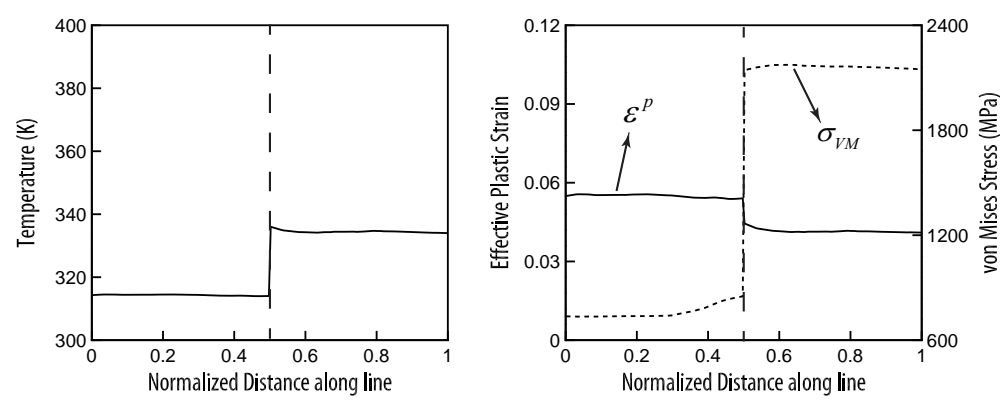

(b)
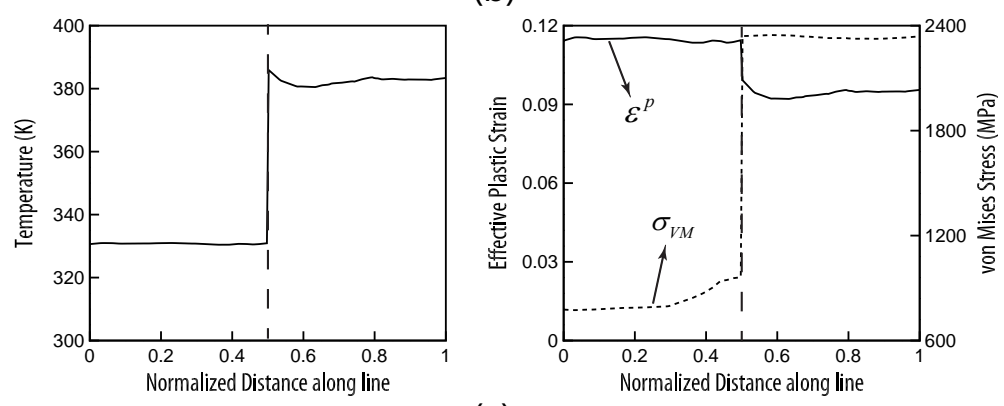

(c)

Fig. 18. Profiles of temperature, effective plastic strain and von Mises stress along a line at (a) $1.5 \%$ strain (stage I), (b) $5 \%$ strain (stage II) and (c) 10\% strain (stage III).

favorably orientated for plastic slip, experience higher adiabatic heating due to the locally higher stress states. The adiabatic simulations should ensure that the local temperature does not exceed the melting temperature of the material during the course of deformation. In the simulations carried out in this paper, the local temperature did not exceed the melting temperature of the material ( $\sim 1950 \mathrm{~K}$ for Ti alloys).

The work in this paper is focused on showing the validity of the novel constitutive models in predicting deformation at a range of strain-rates. However, it paves the way for modeling failure in Ti alloys through the development of physics-based criteria for nucleation of adiabatic shear bands, a precursor to the material failure. 


\section{ACKNOWLEDGMENTS}

This work has been supported by the Army Research Office through grant No. W911NF-12-1-0376 (Program Manager: Dr. A. Rubinstein) and by the Office of Naval research through grant No. N00014-15-1-2040 (Program Manager: Mr. W. Nickerson). The authors would like to thank Dr. Adam Pilchak of AFRL-WPAFB for providing the Ti-7Al samples. Computing support by the Homewood High Performance Compute Cluster (HHPC) and the Maryland Advanced Research Computing Center (MARCC) is gratefully acknowledged. 


\section{References}

Akhtar, A., Teghtsoonian, E., 1975. Prismatic slip in $\alpha$-titanium single crystals. Metall. Mater. Trans. A 6, 2201-2208.

Anahid, M., Samal, M., Ghosh, S., 2011. Dwell fatigue crack nucleation model based on crystal plasticity finite element simulations of polycrystalline titanium alloys. J. Mech. Phys. Solids 59, 2157 - 2176.

Ashby, M., 1970. The deformation of plastically non-homogeneous materials. Phil. Magazine 21, 399-424.

Bhandari, Y., Sarkar, S., Groeber, M., Uchic, M., Dimiduk, D., Ghosh, S., 2007. 3D polycrystalline microstructure reconstruction from FIB generated serial sections for FE analysis. Comput. Mater. Sci. 41, $222-235$.

Brandes, M., 2008. Creep, fatigue, and deformation of alpha and alpha-beta titanium alloys at ambient temperature. Ph.D. thesis. Materials science and engineering. Ohio State University,USA.

Chen, W., Song, B., 2011. Split Hopkinson (Kolsky) Bar: Design, Testing and Applications. Springer Science+Business Media, LLC, Boston, MA.

Cheng, J., Ghosh, S., 2015. A crystal plasticity fe model for deformation with twin nucleation in magnesium alloys. Int. J. Plast. 67, $148-170$.

Cheng, J., Shahba, A., Ghosh, S., 2016. Stabilized tetrahedral elements for crystal plasticity finite element analysis overcoming volumetric locking. Comput. Mech. 57, 733-753.

Clayton, J., 2005. Dynamic plasticity and fracture in high density polycrystals: constitutive modeling and numerical simulation. J. Mech. Phys. Solids 53, 261301.

Culver, R., 1973. Thermal instability strain in dynamic plastic deformation. Metallurgical efects at high strain rates, Plenum press.

Diard, O., Leclercq, S., Rousselier, G., Cailletaud, G., 2005. Evaluation of finite element based analysis of $3 \mathrm{~d}$ multicrystalline aggregates plasticity: Application to crystal plasticity model identification and the study of stress and strain fields near grain boundaries. Int. J. Plasticity 21, $691-722$.

F., R., P., E., T.R., B., Raabe, D., 2010. Crystal plasticity finite element methods: In materials science and engineering. Wiley-VCH Verlag GmbH.

Ghosh, S., Bhandari, Y., Groeber, M., 2008. CAD-based reconstruction of 3D polycrystalline alloy microstructures from FIB generated serial sections. Computer-Aided Design 40, 293 - 310.

Groeber, M., Ghosh, S., Uchic, M., Dimiduk, D., 2007. Development of a robust 3d characterization-representation framework for modeling polycrystalline materials. JOM J. Miner., Met. Materials Soc. 59, 32-36.

Groeber, M., Ghosh, S., Uchic, M., Dimiduk, D., 2008a. A framework for automated analysis and simulation of 3D polycrystalline microstructures: Part 1: statistical characterization. Acta Mater. 56, 1257-1273.

Groeber, M., Ghosh, S., Uchic, M., Dimiduk, D., 2008b. A framework for automated analysis and simulation of 3D polycrystalline microstructures. part 2: Synthetic structure generation. Acta Mater. 56, 12741287. 
Groeber, M., Jackson, M., 2014. DREAM.3D: A digital representation environment for the analysis of microstructure in 3D. Integr. Mater. Manuf. Innov. 3.

Hasija, V., Ghosh, S., Mills, M.J., Joseph, D.S., 2003. Deformation and creep modeling in polycrystalline Ti6Al alloys. Acta Mater. 51, 4533-4549.

Hopkinson, B., 1914. A method of measuring the pressure produced in the detonation of high explosives or by the impact of bullets. Phil. Trans. R. Soc London A213, 437-456.

Hopkinson, B., 1949. An investigation of the mechanical properties of materials at very high rates of loading. Phil. Trans. R. Soc London B62, 676-700.

Huskins, E., Cao, B., Ramesh, K., 2010. Strengthening mechanisms in an AlMg alloy. Mater. Sci. Eng. A 527, 1292 - 1298.

Khan, A., Suh, Y., Kazmi, R., 2004. Quasi-static and dynamic loading responses and constitutive modeling of titanium alloys. Int. J. Plasticity 20, $2233-2248$.

Knezevic, M., Al-Harbi, H., Kalidindi, S., 2009. Crystal plasticity simulations using discrete fourier transforms. Acta Mater. 57, 1777 - 1784.

Lauridsen, E., Schmidt, S., Nielsen, S., Margulies, L., Poulsen, H., Jensen, D., 2006. Non-destructive characterization of recrystallization kinetics using three-dimensional x-ray diffraction microscopy. Scripta Mater. 55, 51-56.

Lebensohn, R., 2001. N-site modeling of a 3d viscoplastic polycrystal using fast fourier transform. Acta Mater. 49, 2723 - 2737.

Lebensohn, R., Kanjarla, A., Eisenlohr, P., 2012. An elasto-viscoplastic formulation based on fast fourier transforms for the prediction of micromechanical fields in polycrystalline materials. Int. J. Plast. 3233, 59 - 69.

Li, H., Mason, D., Bieler, T., Boehlert, C., Crimp, M., 2013. Methodology for estimating the critical resolved shear stress ratios of $\alpha$-phase ti using ebsd-based trace analysis. Acta Mater. 61, 7555 - 7567.

Massey, E., 1951. The kolmogorov-smirnov test for goodness of fit. J. Amer. Statis. Assoc. 46, 68-78.

Medyanik, S., Liu, W., Li, S., 2007. On criteria for dynamic adiabatic shear band propagation. J. Mech. Phys. Solids 55, 1439 - 1461.

Mood, A., Graybill, F., Boes, D., 1974. Introduction to the Theory of Statistics, 3rd edition. McGraw-Hill.

Ogi, H., Kai, S., Ledbetter, H., Tarumi, R., Hirao, M., Takashima, K., 2004. Titaniums high-temperature elastic constants through the hcpbcc phase transformation. Acta Mater. 52, 2075-2080.

Osovski, S., Rittel, D., Venkert, A., 2013. The respective influence of microstructural and thermal softening on adiabatic shear localization. Mech. Mater. 56, $11-22$.

Pilchak, A., 2013. Fatigue crack growth rates in alpha titanium: Faceted vs. striation growth. Scripta Mater. 68, $277-280$.

Pilchak, A., 2015. Unpublished Report.

Rittel, D., Wang, Z.G., Merzer, M., 2006. Adiabatic shear failure and dynamic stored energy of cold work. Phys. Rev. Lett. 96, 075502. 
Roters, F., Eisenlohr, P., Hantcherli, L., Tjahjantoa, D., Bieler, T., Raabe, D., 2010. Overview of constitutive laws, kinematics, homogenization and multiscale methods in crystal plasticity finite-element modeling: Theory, experiments, applications. Acta Mater. 58, 1152-1211.

Rowenhorst, D., Voorhees, P., 2005. Measurements of the grain boundary energy and anisotropy in tin. Metall. Mater. Trans. A 36A, 2127-2135.

Russ, J., Dehoff, R., 1999. Practical streology, 2nd edition. Plenum press.

Shade, P., 2015. Unpublished report.

Shahba, A., Ghosh, S., 2016. Crystal plasticity fe modeling of ti alloys for a range of strain-rates. part I: A unified constitutive model and flow rule. Int. J. Plast. Unpublished.

Simulation Modeling Suite, $2015 . \quad$ Simmetrix Inc. http://www.simmetrix.com.

Tanaka, T., Conrad, H., 1972. Deformation kinetics for $10 \overline{1} 011 \overline{2} 0$ slip in titanium single crystals below $0.4 t_{m}$. Acta Metal. 20, $1019-1029$.

Thomas, J., Groeber, M., Ghosh, S., 2012. Image-based crystal plasticity FE framework for microstructure dependent properties of Ti6Al4V alloys. Mater. Sci. Eng. A 553, 164-175.

Williams, J., Baggerly, R., Paton, N., 2002. Deformation behavior of HCP Ti-Al alloy single crystals. Metall. Mater. Trans. A 33, 837850.

Zhang, C., Li, H., Eisenlohr, P., Liu, W., Boehlert, C., Crimp, M., Bieler, T., 2015. Effect of realistic 3d microstructure in crystal plasticity finite element analysis of polycrystalline ti-5al-2.5sn. Int. J. Plasticity 69, $21-35$. 